\title{
Comparison of Plasma and Cerebrospinal Fluid Proteomes Identifies Gene Products Guiding Adult Neurogenesis and Neural Differentiation in Birds
}

Eleni Voukali ( $\sim$ voukalie@natur.cuni.cz)

Charles University

Nithya Kuttiyarthu Veetil

Charles University

Pavel Němec

Charles University

Pavel Stopka

Charles University

Michal Vinkler

Charles University

\section{Research Article}

Keywords: Cerebrospinal fluid (CSF), brain homeostasis, nLC-MS/MS, Melopsittacus undulatus

Posted Date: January 5th, 2021

DOI: https://doi.org/10.21203/rs.3.rs-138227/v1

License: (9) This work is licensed under a Creative Commons Attribution 4.0 International License.

Read Full License

Version of Record: A version of this preprint was published at Scientific Reports on March 5th, 2021. See the published version at https://doi.org/10.1038/s41598-021-84274-x. 


\section{Abstract}

Cerebrospinal fluid (CSF) proteins regulate neurogenesis, brain homeostasis and participate in signalling during neuroinflammation. Even though birds represent valuable models for constitutive adult neurogenesis, current proteomic studies of the avian CSF are limited to chicken embryos. Here we use liquid chromatography-tandem mass spectrometry (nLC-MS/MS) to explore the proteomic composition of CSF and plasma in adult chickens (Gallus gallus) and evolutionarily derived parrots: budgerigar (Melopsittacus undulatus) and cockatiel (Nymphicus hollandicus). Because cockatiel lacks a complete genome information, we compared the cross-species protein identifications using the reference proteomes of three model avian species: chicken, budgerigar and zebra finch (Taeniopygia guttata) and found the highest identification rates when mapping against the phylogenetically closest species, the budgerigar. In total, we identified 483, 641 and 458 unique proteins consistently represented in the CSF and plasma of all chicken, budgerigar and cockatiel conspecifics, respectively. Comparative pathways analyses of CSF and blood plasma then indicated clusters of proteins involved in neurogenesis, neural development and neural differentiation overrepresented in CSF in each species. This study provides the first insight into the proteomics of adult avian CSF and plasma and brings novel evidence supporting the adult neurogenesis in birds.

\section{Introduction}

The central nervous system (CNS) function is regulated by a vivid communication with other tissues. This is also achieved through the cerebrospinal fluid (CSF) that supports the brain cell functions ${ }^{1}$. Being from $\sim 80 \%$ plasma $\left(\mathrm{PL}\right.$ )-derived and $\sim 20 \%$ brain-cell-derived $^{2}$, CSF dynamically reflects both peripheral and CNS physiological processes. Despite the paramount importance of the relationship between blood and CSF, little comparative effort has been made in non-mammalian vertebrates to characterise the two vital fluids integrating processes in and out of the CNS.

There is widespread evidence that the birth and growth of neurons also occurs in adult invertebrate and vertebrate CNS and that these cells add onto existing neuronal networks or replace apoptotic neurons ${ }^{3}$. In addition, several in vitro and in vivo studies have provided evidence that, CSF can promote neurogenesis even in the adult brain ${ }^{4-9}$. In particular, spatial gradients of CSF proteins induced the migration of neuroblasts from the subventricular zone to the olfactory bulb in the adult mouse brain ${ }^{8}$. Also, when rat CSF was applied as a culture medium to neural stem cells and cortical explants, the cells survived without additional supplements, particularly for age-matched CSF samples and tissues ${ }^{9}$. Therefore, increased interest has been raised to study and describe the molecular composition of CSF, especially in humans ${ }^{10-16}$.

Although CSF proteomic composition shares many similarities across species such as rat, human and chicken ${ }^{6,10-12}$, species differ in physiological requirements of their tissues and different species show different neurogenesis rates as well as anatomical features ${ }^{11,17}$. Birds have been proposed as suitable 
models to investigate the molecular basis of the regulation of adult constitutive neurogenesis ${ }^{18}$. Nonetheless, the available avian CSF proteomic analyses have addressed only embryonic chicken CSF. Based on two-dimensional polyacrylamide gel electrophoresis (2D-PAGE) followed by mass spectrometry (MS) or tandem mass spectrometry (MS/MS) methods, the chicken embryonic CSF (eCSF) consists of hundreds of proteins of the extracellular matrix, regulators of osmotic pressure, ion carriers, hormonebinding proteins, regulators of lipid metabolism, and various enzymes and their regulators ${ }^{6,10,11}$. However, the advent of methods such as gel-free-LC/MS for comparative protein analysis has resulted in several advantages over the label-based methods used by the aforementioned studies, including the greater coverage of the proteome ${ }^{19}$.

While the domestic chicken (Gallus gallus, GG, order Galliformes) serves as a basic avian model organism in developmental biology and neuroscience ${ }^{20,21}$, current evidence shows that its neural densities are much lower than in some other, evolutionarily derived birds ${ }^{22,23}$. Importantly, recently, parrots (order Psittaciformes) have been proposed as models for neurobiological research ${ }^{24}$. Similarly to humans, parrots have large brains relative to their body $\operatorname{size}^{25}$, high density of neurons in the forebrain ${ }^{22}$, exhibit outstanding cognitive skills such as object permanence 26,27 and tool use ${ }^{26,28}$, cooperative problem solving ${ }^{29}$, complex social organization ${ }^{30}$, learning of vocalizations through cultural transmission ${ }^{31,32}$, and some species are on par with great apes in number of cognitive domains ${ }^{33-35}$. Furthermore, despite their high metabolism, they show extended developmental periods ${ }^{36}$ and longevity 24,37 .

In the present study, we focus for the first time on avian adult CSF and PL proteomes, comparing the domestic chicken to two parrot species: the budgerigar (Melopsittacus undulatus, $\mathrm{MU}$ ) and the cockatiel (Nymphicus hollandicus, NH), representing two distinct parrot clades (Psittacoidea and Cacatuoidea, respectively ${ }^{38}$ ). Since the cockatiel genome is not presently available, a condition that may affect the peptide/protein identification, we first test the consistency of the proteomic annotation by comparing the MS/MS identification success rates after mapping all spectra against the reference proteomes of three model avian species: chicken, budgerigar and zebra finch (Taeniopygia guttata). Second, using the quality-filtered and consistent data, we describe the proteomic profiles of PL and CSF in the three bird species, searching for the pathways common to differentiate the avian PL and CSF proteomes.

\section{Results}

\section{Reference selection for proteome mapping.}

We mapped each chicken, budgerigar and cockatiel CSF and PL sample spectra against the chicken, zebra finch and budgerigar reference proteomes (Fig. 1a). The total numbers of spectra, numbers of unique spectra and numbers of the identified peptide sequences obtained for each mapping combination are shown in Supplementary Table S1, along with the total numbers of unique proteins identified for each data set before and after filtering. The list of proteins represented in individual samples together with their 
abundances and the ortholog gene names corresponding to each identified protein ID is also provided in Supplementary Table S1. We found that a large proportion $(25.1 \%, 35 \%$ and $41.1 \%$ for chicken, budgerigar and cockatiel, respectively) of orthologous gene IDs overlapped when mapped against chicken, zebra finch and budgerigar reference proteomes in either CSF or PL, being consistent for all avian species examined in this study (Fig. 1b). However, part of the proteins remained unidentified when the CSF and $\mathrm{PL}$ spectra were mapped against either the same species proteome or the budgerigar proteome in the case of cockatiel. Regarding chicken spectra, about 7.2\% (37) out of the total proteins identified for chicken were not identified when mapped against the chicken reference proteome but found when mapped against zebra finch and budgerigar references; for the budgerigar, 18\% (125) of identities were missed when mapped against budgerigar genome, but detected when mapped against the zebra finch or chicken; and for the cockatiel, $13.8 \%$ (70) of identities were missed when mapped against the budgerigar proteome, but detected when mapped against the zebra finch and/or chicken. The list of these genes is included in Supplementary Table S1 and contains, among others, members of multigene families such as histones, and myoglobins. A Gene Ontology (GO) classification of the biological functions for each subset of these missing proteins is shown in Fig. S1 in Supplement. The most represented biological functions of these missing proteins were: 1$)$ Cellular processes $(61.1,47.7,53.3 \%$ of the missing proteins in $\mathrm{GG}, \mathrm{MU}$ and $\mathrm{NH}$, respectively) including the cell communication, cellular component organization, developmental process, homeostasis, metabolic process, response to stimulus, export from cell, movement of cell or subcellular component, protein folding and signal transduction; 2) Biological regulation of molecular functions $(27.8,19.3,28.9 \%$ of the missing proteins in $\mathrm{GG}, \mathrm{MU}$ and $\mathrm{NH}$, respectively); and 3) Metabolic processes $(27.8,6.4,35.6 \%$ of the missing proteins in GG, MU and NH respectively) including ATP metabolic process, biosynthetic process, catabolic process, cellular metabolic process, nitrogen compound metabolic process, organic substance metabolic process, oxidationreduction process, primary metabolic process and small molecule metabolic process. Since mappings from multiple annotations could not be combined and a higher proportion of gene products were exclusively identified when the proteomes were mapped against the same species reference proteome (chicken and budgerigar) or a closely related one (cockatiel), we selected only these datasets for further analysis and focused mainly on consistently represented proteins.

\section{Blood PL and CSF proteomes description.}

In total, after filtering, we identified 464 proteins represented in the chicken CSF proteome, 586 proteins in the budgerigar CSF proteome and 437 proteins in the cockatiel CSF proteome. The PL proteome consisted of 304 proteins in the chicken, 478 in the budgerigar and 310 in the cockatiel. From these, 109 proteins (11.3\%) were common to all species (GG, MU, NH) and all sample types (PL or CSF) (Fig. 2a), while a large number of proteins varied even between individual samples of the same tissue and species (Supplementary Fig. S2). The detailed protein coverage of each dataset is shown on Supplementary Fig. S3. We then defined the core proteomes, that is, proteins represented in all conspecific individuals for each fluid. The complete list of the proteins and abundances of the CSF and PL core proteomes for the three species is tabulated in the Supplementary Table S2. Considering the list of proteins detected across 
all individuals across all species in the particular sample types, we identified 115 common proteins in the CSF (22.3\%, Fig. 2b), 53 common proteins in the PL (15.8\%, Fig. 2c), and 40 proteins overlapping between CSF and PL (Fig. 2 d). The CSF core proteome consisted of 344 proteins $(71.22 \%$ of the total proteins detected across all individuals) in chicken, 269 proteins (41.97\%) in budgerigar and $274(56.92 \%)$ proteins in cockatiel. The PL core proteome consisted of 173 proteins $(55.27 \%)$ in chicken, 227 proteins $(45.31 \%)$ in budgerigar and 124 proteins $(38.87 \%)$ in cockatiel.

We ranked the shared identified proteins according to their normalized logarithmic abundance means. There was a considerable inter-individual variability in the expression of the lowly abundant proteins in both PL and CSF. In contrast, among the highly abundant proteins, the protein composition appears relatively consistent in both fluids between the three species, parrot species being more similar (Fig. 3). Among the $2 \%$ most abundant proteins identified in both the fluids and across all the species belongs namely albumin (ALB), ovotransferrin (TF), alpha 2 macroglobulin (A2M), transthyretin (TTR), vitronectin (VTN) and apolipoproteins (APOA1, APOA4).

In general, the proteins commonly represented in CSF and blood PL were involved in metabolic and signalling pathways and most of their functions are common to CSF and plasma (Fig. 4). As expected, CSF proteins are mostly extracellular components expressed into the extracellular space. The complete results of the pathway enrichment analysis are shown in Supplementary Table S3.

\section{Comparison of the plasma and CSF proteomes.}

Raw and normalised data were inspected by their log2 distributions and correlation of their principal component 1 of the shared genes. After data normalization, the distributions showed almost no variation between the samples and their first principal component positively correlated with the first principal component of raw data (Supplementary Fig. S4). Principal component analyses (PCA) of CSF and PL protein abundances showed that the two fluids form separate clusters for all three studied species (Supplementary Fig. S5). For chicken, the first two principal components related to fluid type explained $84.76 \%$ of the total variance; for budgerigar $70.22 \%$ and cockatiel $74.21 \%$, indicating that CSF and PL proteomes are distinguishable in these species. The exploration of the differentially represented proteins in CSF and PL proteomes in GG identified 413 significantly differentially abundant proteins, including 49 under-represented proteins and 289 over-represented proteins in CSF compared to PL with false discovery rate (FDR) adjusted $p$-value $<0.05$ and fold change cut-off $\geq 2$. Similarly, 332 proteins were significantly differentially represented in MU CSF compared to PL proteome, including 99 under-represented and 224 over-represented proteins (FDR adjusted p-value $<0.05$, fold change cut-off $\geq 2$ ) and, for $\mathrm{NH}$, the analysis revealed 289 differentially represented proteins, 46 under-represented and 206 over-represented in CSF compared to PL (FDR adjusted $p$-value, fold change cut-off $\geq 2$ ). The fold change differences in the common proteins across species are shown in volcano plots at Fig. $5 \mathrm{a}$. The fold change differences in all differentially represented proteins for each species are shown in the Supplementary Fig. S6. Detailed information on these differentially represented proteins is provided in the Supplementary Table S4. The 
differentiation between protein content of CSF and PL was further indicated based on hierarchical clustering that we used to visualise the groups of differentially represented proteins in either CSF or PL in all the three studied avian species (Fig. 5b).

We found that 72 proteins over-represented in CSF compared to PL overlap between GG, MU or NH, belonging to various functional modules such as cell-adhesion proteins, growth factors, subunits of calcium channels etc. Interestingly, four commonly shared CSF proteins (LCAT, phosphatidylcholine-sterol acyltransferase; MCFD2, multiple coagulation factor deficiency protein 2; PRDX1, peroxiredoxin-1) are under-represented in the CSF of parrots but over-represented in chicken. We did not identify any proteins over-represented in the CSF of chicken but under-represented in parrots. STRING analysis of the 72 commonly over-represented proteins in CSF of all species indicates a network of protein interactions, containing 72 nodes and 199 edges (Fig. 6a). The GO Biological function terms related to this proteinprotein interaction (PPI) network indicate developmental and neogenesis processes. The same gene set was visualised as a hierarchical clustering tree using ShinyGO (Fig. 6b).

This is consistent with the result of the gene set enrichment analysis (GSEA) based on GO Biological functions classification and pathway enrichment in CSF compared to plasma that revealed differential representation in proteins involved in the neural function. Enriched pathways in plasma were mostly related to immune functions. Supplementary Table 55 shows the complete list of the significantly enriched pathways at nominal p-value $<0.05$ and FDR $<0.25$ in $\mathrm{CSF}$ and $\mathrm{PL}$, and Supplementary Figs. S710 the graphical representation of their gene overlap. The GSEA analysis revealed several gene sets linked to neurogenesis, neural development and differentiation which were preferentially expressed into CSF and shared across the species (Fig. 7). Figure 8 shows the nominal enrichment scores for GO term Neurogenesis and the genes implicated for each species.

\section{Discussion}

CSF connects the bloodstream with the CNS and reflects the physiology of the brain. Here we have described the adult CSF and PL protein composition in three avian species (chicken, budgerigar, and cockatiel) using gel-free nLC-MS/MS. First, we have confirmed that the phylogenetically closest avian reference proteome yields the highest efficacy in protein identification success rates, although a proportion of proteins $(7.2 \%, 18 \%$ and $13.8 \%$ for $\mathrm{GG}, \mathrm{MU}$ and $\mathrm{NH}$, respectively) was mapped only using other references. In total, following the data filtering, we identified in the CSF and PL 483 proteins in the chicken, 641 proteins in the budgerigar and 458 proteins in the cockatiel. GO pathway analyses of the core proteomes revealed components of several signalling and metabolic processes such as regulation of peptidases, synapse organisation, regulation of IGF transport and uptake by IGFBPs in CSF and blood coagulation, complement activation and glycolysis in PL. Comparative analysis of the proteomes derived from the two biological fluids showed that the CSF and PL are clearly distinguishable in terms of their protein content. In CSF, the pathways involved in neurogenesis, and neural development and differentiation are over-represented when compared to blood plasma. 
Current research increasingly adopts investigation in diversified taxa lacking complete and annotated genomes and proteomes. In this study, we investigated also the cockatiel, a species currently lacking a genome sequence. We found that by cross-species protein identification using the sequence database from its evolutionarily closest relative, the budgerigar, we could identify 458 proteins represented in the CSF and PL. This is more than if other reference genomes were used (chicken, 327; zebra finch, 291). This trend was consistent with our findings in other species included in this study. Interestingly, however, some proteins were identified only when mapped against the other mapping combinations. Quantitative proteomics of unsequenced organisms is still a challenge and, when de novo sequencing of the unidentified proteins is unavailable, the reference proteome of a close-related phylogenetically species is utilised to identify the proteome in question. An analogous approach has been used to characterise the proteome of unsequenced micro-organisms and Xenopus laevis microtubule-associated proteome ${ }^{39,40}$, but this is the first study reporting a paradigm of cross-species quantitative proteomics in diverse avian taxa.

We found more proteins in CSF compared to plasma, a finding which was consistently found in all avian species in this study and similar to what has been previously reported for humans in paired samples 41,42 . In a cohort of more than 100 individuals, Dayon et al. identified 790 and 422 proteins in parallel CSF and $\mathrm{PL}$ samples respectively with an overlap of 255 proteins shared by both fluids ${ }^{41}$. In a previous study, a maximal protein set of 3,081 and $1,050 \mathrm{CSF}$ and PL proteins was identified respectively with 877 proteins common in both fluids from a group of 21 healthy individuals ${ }^{42}$. The reasons for the differences in the number of protein identifications between these two studies could be attributed in different sample preparation approaches and detection techniques. Thus, although the protein concentration is likely higher in $\mathrm{PL}$ compared to $\mathrm{CSF}^{43}$, the variety of proteins is greater in the CSF compared to PL, being enriched from ca. $20 \%$ with proteins expressed in the choroid plexus and brain ${ }^{44}$. Blood and serum comprise receptor ligands, immunoglobulins, tissue leakage products, aberrant secretions and proteins that are extraneous to the animal. Many blood proteins are synthesized in liver ${ }^{45}$. Although quality of CSF collection can influence the protein content observed, the CSF concentration of CNS-derived proteins remains unaffected following contamination of the fluid with blood ${ }^{46}$. In the present dataset, we found the presence of haemoglobin subunits and carbonic anhydrase that can be attributed to some minor blood contamination ${ }^{47}$. Yet, like in Smith et al. ${ }^{13}$, although haemoglobin subunits were found amongst the most CSF abundant proteins, our clustering analysis clearly differentiated CSF and plasma samples in all studied individuals and $58.5 \%$ of the shared proteins across species, such as gamma-enolase, neuronal growth regulator 1 or neural cell adhesion molecule 1, could be identified selectively in CSF.

Using gel-based techniques and mass spectrometry, the proteomes of eCSF from rat, chicken and human have been previously described to elucidate the molecular composition of CSF and its biological role during embryonic neurogenesis ${ }^{10-12,48}$. Human eCSF contained 188 proteins, $44 \%$ of those also present in rat eCSF. Functional analysis revealed protease inhibitors, extracellular matrix proteins, and transport proteins as well as signalling and intracellular proteins ${ }^{12}$. Comparing the data available for human eCSF with our results in birds, $39 \%, 50 \%$ and $47 \%$ of the proteins overlap with adult CSF from chicken, 
budgerigar and cockatiel, respectively. In chicken embryos investigated at the developmental stage $\mathrm{HH} 24$, using 2D-PAGE, 26 proteins were identified, related to the extracellular matrix, regulation of the osmotic pressure and metal transport, cell survival, transport of retinol and vitamin D as well as mitogen-activated protein (MAP) kinase activators, antioxidant and antimicrobial proteins, intracellular proteins and some unknown proteins. We found that $50 \%$ of these proteins were included also in our data. Many of these proteins regulate the development in systems other than CNS, while others are altered during neurodegeneration ${ }^{10,11}$.

LC-based methods allowed the identification of a dramatically increased number of proteins in CSF. Most recently, using LC-MS/MS after abundant protein depletion, Macron et al. identified 3,174 proteins in a commercial pool of "normal" human CSF samples ${ }^{15}$. However, expectedly, interindividual variation is accountable for identification of many of the rare proteins. For example, out of 2,615 CSF proteins, only $20 \%$ of the identified proteins were shared by all six healthy subjects ${ }^{16}$. In a combined dataset from three studies the full human CSF proteome of 4,814 proteins was revealed, but only about $40 \%$ of these proteins were common. We found that about a half of the proteins identified in either plasma or CSF in each species showed a high degree of inter-individual variability, while the other half was relatively consistently represented across all individuals. In our study we have shown that 71.22 and $55.27 \%$ (GG), 41.97 and $45.31 \%(\mathrm{MU})$, and 56.92 and $38.87 \%(\mathrm{NH})$ proteins identified for CSF and PL respectively were common to all individuals within species and only 193 proteins were common across all three species. Thus, the number of proteins that are individually specific is high. The reasons for this variation, however, remain unclear ${ }^{42,49,50}$. The molecular variations in CSF composition can be possibly increased by posttranslational modifications, age, blood contamination ${ }^{47}$ and circadian rhythm ${ }^{51}$.

Our comparative analysis shows that CSF and plasma were distinguishable based on their proteome compositions. From the proteins over-represented in CSF compared to plasma, such as homologs of neuronal growth regulator 1 (NEGR1) and reelin $(R E L N), 72$ were shared across all the three avian species. A PPI network constructed based on these proteins, as well as the enrichment analysis of biological functions revealed namely representation of pathways associated with cell adhesion, nervous system development and neurogenesis. In addition, using GSEA, we indicated in CSF enrichment of pathways involved in neural functions across the studied species. In addition to those revealed by differential analysis, the CSF samples shared several gene products related to neurogenesis for example neogenin 1 (NEO1), neurofascin (NFASC), neuromodulin (GAP43), or pigment epithelium-derived factor (SERPINF1). Our results suggest that adult neurogenesis occurs in all the avian species studied. Previous reports have stressed the importance of various growth factors present in CSF which bind to receptors located on the apical membrane of cortical progenitor cells and promote the development and growth of neural stem cells and cortical explants ${ }^{9,52}$. In this study, we identified proteins such as 14-3-3 proteins (YWHAG, $Y W H A Z$ ) and fibroblast growth factor receptor 1 (FGFR1-4) participating in the fibroblast growth factor pathway ${ }^{53}$, molecules interacting with the $\mathrm{Wtn}{ }^{9}$ pathway such as the Wtn inhibitor Dickkopf-related protein $3(D K K 3)$ and cadherins $(C D H 13, C D H 2, C D H 6, C D H 4)$, amyloid beta A4 protein precursor $(A P P)^{12}$, insulin-like growth factor II (IGF2) and insulin-like growth factor-binding proteins (IGFALS, IGFBP2, 


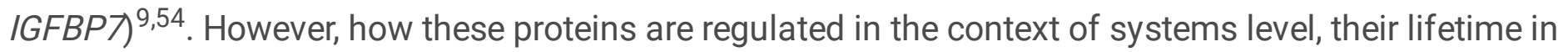
$\mathrm{CSF}$, as well as the contribution of interindividual variability requires further investigation.

In vitro studies of adult human CSF treatment in neural stem cells showed that adult human CSF promoted gliogenesis, but not neurogenesis ${ }^{55,56}$. Despite ontogenetic differences in CSF protein composition in its ability to induce neurogenesis, the neurogenic potential is preserved in the adult mammalian brain and can be activated only by external stimuli including exercise, signalling molecules following injury or eCSF 6,57 . In contrast, in birds, neuronal cell proliferation occurs in the subventricular zone of the lateral ventricle, and migration to widespread regions of telencephalon follows ${ }^{58}$. Immunohistochemical studies have demonstrated adult neurogenesis in the telencephalon and other brain regions of canaries ${ }^{59}$, marsh tits ${ }^{60}$, ring doves ${ }^{61}$, chicken $^{62}$, pigeons $s^{63,64}$, parrots ${ }^{65}$, ostrich and $\mathrm{emu}^{66}$. Although it is well established that factors such as age, environmental complexity, seasonal variation, hormones, stress and social complexity influence its intensity ${ }^{18}$, the functional role of adult neurogenesis in birds remains unknown. It has been proposed to be associated with continuous learning. In adult mammals, the proliferative hot spots may disappear altogether ${ }^{58}$, but comparative approaches between birds and mammals have elucidated similarities on the origin of neurons, the phenotype and proliferative mechanisms of stem cells and the migration and differentiation of neurons as well as differences such as the spatial distribution of adult-born neurons, their mode of migration and phenotypic diversity $3,65,67$. We found that chicken and parrots share common proteins and pathways associated with the modulation of neuronal cell proliferation and plasticity in CSF. Although our identification of these gene sets and pathways in birds serves only as an initial screening and subsequent experimental verification is required, our study forms an essential reference for further molecular analysis.

\section{Conclusion}

The advent of the proteomic techniques has allowed new insights into molecular processes driving the CNS function, with research in CSF forming its cornerstone. For the first time, we concurrently explored in birds the proteomes of two main biological fluids, PL and CSF. Importantly, we focused on multiple species, the chicken and two parrot species, the budgerigar and cockatiel, investigating adult individuals. The proteins over-represented in CSF were mainly classified into functional pathways involved in neural function, providing a novel molecular evidence for adult neurogenesis in birds. Consistently with histological evidence for adult neurogenesis in birds, understanding the molecular components involved in this process is essential for designing future experimental work in this system.

\section{Methods}

\section{Sample collection.}

CSF and PL samples were collected from a total of seven chicken, five budgerigars and five cockatiels. The animals were originated from legal imports for the pet trade (Prague, Czech Republic) who confirmed 
their status as healthy, adult birds and were kept in our animal facility (accreditation No. 37428/2019MZE-18134). The animals were euthanised by $\mathrm{CO}_{2}$ and mounted on a stereotaxic instrument (Stoelting Co., Illinois, USA). After incision in the dorsal neck region and exposure of the skull occipital region, a needle of a $0.5 \mathrm{ml}$ sterile insulin syringe was inserted into cisterna magna ${ }^{68}$. Approximately $3-10 \mu \mathrm{l}$ of clear, colourless CSF was collected per animal and stored at $-80^{\circ} \mathrm{C}$ until analysis preparation. Blood was extracted from the carotid artery and PL obtained by centrifugation $(14,000 \mathrm{~g})$ was stored at $-80^{\circ} \mathrm{C}$, until further use. Experimental design involving animals was performed by accredited researchers (EV: CZ03814, MV: CZ02568). The research was approved by the Ethical Committee of Charles University, Faculty of Science (permits MSMT-1373/2016-4 and MSMT-30397/2019-5) and was carried out in accordance with the current laws of the Czech Republic and European Union and in compliance with the ARRIVE guidelines.

\section{Protein extraction and precipitation.}

The ProteoSpin detergent-free total protein isolation kit (Norgen Biotek, Thorold, ON, Canada) was used for isolation and purification of the total protein content from CSF and PL according to the manufacturer's instructions and then frozen at $-20^{\circ} \mathrm{C}$. Protein precipitation was performed using acetone $\left(1 / 5\right.$, acetone/protein) at $-20^{\circ} \mathrm{C}$ for $1 \mathrm{~h}$. All precipitated samples were centrifuged at $14,000 \mathrm{~g}$ and $4{ }^{\circ} \mathrm{C}$ for $15 \mathrm{~min}$. After centrifugation, the supernatant was discarded, the samples dried for $30 \mathrm{~min}$ at $37^{\circ} \mathrm{C}$ and were cleaved with trypsin (i.e., $1 / 50$, trypsin/protein) at $37^{\circ} \mathrm{C}$ overnight before mass spectrometry analysis.

\section{nLC - MS/MS Analysis.}

Nano reversed-phase columns were used to elute peptide cations using a previously described method ${ }^{69}$. The eluting peptide cations were converted to gas-phase ions by electrospray ionization and analysed on a Thermo Orbitrap Fusion mass spectrometer (Q-OT-qIT, Thermo). Survey scans of peptide precursors from 350 to $1400 \mathrm{~m} / \mathrm{z}$ were performed at $120 \mathrm{~K}$ resolution (at $200 \mathrm{~m} / \mathrm{z}$ ) with a $5 \times 105$ ion count target. Tandem MS/MS was performed by isolation at $1.5 \mathrm{Th}$ with the quadrupole, high-energy collision dissociation (HCD) fragmentation with a normalized collision energy of 30 and rapid scan MS analysis in the ion trap. The MS/MS ion count target was set to 104 and the max injection time was $35 \mathrm{~ms}$. Only those precursors with a charge state of 2 - 6 were sampled for MS/MS. The dynamic exclusion duration was set to $45 \mathrm{~s}$ with a 10-ppm tolerance around the selected pre-cursor and its isotopes. Monoisotopic precursor selection was turned on and the instrument was run at top speed with $2 \mathrm{~s}$ cycles.

\section{Proteome data analysis.}

All data were collected and quantified using MaxQuant software version 1.6.10.43 ${ }^{70}$. False discovery rate (FDR) was set to $1 \%$ for identification of all peptides and proteins. We set a minimum peptide length of 
seven amino acids. The Andromeda search engine was used for the MS/MS spectra search against the chicken Gallus gallus UniProt reference proteome (downloaded on March 2020, containing 27,540 entries), budgerigar Melopsittacus undulatus UniProt reference proteome (downloaded on February 2020, containing 23,704 entries) and zebra finch Taeniopygia guttata UniProt reference proteome (downloaded on February 2017, containing 23,455 entries), with all duplicates removed. Enzyme specificity was set as C-terminal to Arg and Lys, also allowing cleavage at proline bonds ${ }^{71}$ and a maximum of two missed cleavages. Dithiomethylation of cysteine was selected as a fixed modification and $\mathrm{N}$-terminal protein acetylation and methionine oxidation as variable modifications. Quantifications were performed with the label-free quantification algorithms using a combination of unique and razor peptides ${ }^{70}$. The mass spectrometry proteomics data have been deposited to the ProteomeXchange Consortium via the PRIDE ${ }^{72}$ partner repository with the dataset identifier PXD021633.

\section{Mapping approaches.}

Annotated reference proteomes are presently available for the chicken ${ }^{73}$ and the budgerigar ${ }^{74}$, but not for the cockatiel. Thus, mapping cockatiel reads on its own reference proteome was not possible. To estimate the effect of this condition, we compared the MS/MS identification success rates of chicken, budgerigar and cockatiel proteomes, each mapped against three applicable avian reference proteomes, the chicken, budgerigar and zebra finch. We performed the following data filtering to eliminate lowscoring spectra: from further analysis we excluded all the unlabelled peptides, peptides only identified by site or reverse, all contaminants, proteins identified by $<2$ peptides and all proteins occurring in less than $1 / 3$ of the samples for each group. For the purpose of comparison, chicken ortholog gene codes were assigned to all identified proteins using the database OrthoDB $(\mathrm{v} 10.1)^{75}$. When the gene code was not possible to retrieve, the UniProt protein ID was used.

For protein classification, we used the online Protein Analysis Through Evolutionary Relationships (PANTHER) library ${ }^{76}$. To find overrepresented Gene Ontologies (GOs ${ }^{77}$ in the core proteomes of CSF and $\mathrm{PL}$, we launched the g:profiler against the chicken reference list (all genes in the database) and the annotation data sets of the Gene Ontology and biological pathways (KEGG, REACTOME) ${ }^{78-80}$. The Benjamini-Hochberg FDR threshold was set to 0.05 of all identified proteins in PL and CSF of chicken, budgerigar and cockatiel.

\section{Statistical analysis.}

The statistical and bioinformatic analysis conducted in the R software ${ }^{81}$, Microsoft Excel and Perseus software platform (http://www.perseus-framework.org; Max Planck Institute of Biochemistry, Martinsried, Germany). The Venn diagrams were prepared using Biovenn ${ }^{82}$ and Jvenn ${ }^{83}$. In order to remove minor technical variation between samples, the abundances of the identified proteins were previously normalised using the Variance stabilization normalization (VSN) method ${ }^{84,85}$ and DEP 1.12.0 R package. 
Missing data were imputed using random draws from a manually defined left-shifted Gaussian distribution applying the default settings of Perseus software. PCA was performed to examine the variation between the PL and CSF fluids in these species using Perseus and R. Significantly differentially represented proteins were identified by a Student's t-test corrected by the Permutation Based FDR (FDRadjusted $p$-value $<0.05$ ). In order to visualise biological protein groups clustered together in either CSF or $\mathrm{PL}$, hierarchical cluster analysis was performed in Perseus using default parameters (Euclidean distance with average linkage, 300 clusters, 10 iterations, 1 restart and k-means pre-processing for both row and column tree). To indicate the proteins significantly differentially represented in CSF and PL in the three studied species, a Volcano Plot analysis was performed in Perseus (unpaired Student's T-test, S0 = 2, permutation-based FDR) and R. In the volcano plot, protein intensity fold-change between the fluids is represented as $\log _{2}$. The Retrieval of Interacting Genes (STRING 11.0; http://string-db.org) ${ }^{86}$ web-tool was used to analyse and construct the PPI network of the common group of proteins across the three species that were over-represented in CSF compared to PL (adjusted p-value cut off $(F D R)<0.05$, identified by the t-test analysis described above). The interaction sources included experimental data and significant PPIs were considered those with a combined score $>0.4$ and annotations from human (Homo sapiens). For the network visualization, also using annotations from Homo sapiens, we used ShinyGO (v0.61) ${ }^{87}$ online tool to determine the $\mathrm{GO}$ terms of biological functions which are significantly overrepresented in CSF compared to blood PL in the same set of common gene products over-represented in the CSF compared to PL. GSEA was also performed. All proteins were ranked based on the association between their abundance and the class distinction (CSF or PL), using annotations from Homo sapiens (Human Gene Symbol with Remapping MSigDB.v7.2). We used the GOs analysis of biological processes, nominal pvalue $<0.05$ and adjusted $p$-value $<0.25$, as previously suggested. An FDR of $<25 \%$ is recommended for discovering candidate gene sets to be further validated as a result of future research ${ }^{88}$.

\section{Declarations}

\section{Data Availability Statement}

The mass spectrometry proteomics data have been deposited to the ProteomeXchange Consortium via the PRIDE ${ }^{72}$ partner repository with the dataset identifier PXD021633.

\section{Acknowledgements}

We are thankful to Karel Harant and Pavel Talacko from the Mass Spectrometry and Proteomics Service Laboratory, Faculty of Science, Charles University for performing the nLC-MS/MS run and to Romana Stopková for her guidance with protein purification.

We are also grateful to Kathryn Brown for language editing. This study was supported by the Charles University (grants Nos. PRIMUS/17/SCI/12 and UNCE 204069), by the Czech Science Foundation (grant No. P502/19-20152Y), by the Ministry of Education, Youth and Sports of the Czech Republic (Inter-COST 
grant No. LTC18060 and through the Institutional Research Support No. 260571/2020) and the MICOBION project funded from EU H2O20 (No 810224).

\section{Author contributions}

The authors confirm contribution to the paper as follows: study conception and design: MV, EV; data collection: EV, MV; protein extraction and precipitation with input from: EV, PS; analysis and interpretation of results: EV, NK; statistical support in analysing the data with input from: PS; draft manuscript preparation and critical revision for important intellectual content: EV, MV. PN co-supervised the tissue sampling. All authors reviewed the results and approved the final version of the manuscript.

\section{Competing Interests Statement}

The authors declare no competing interests.

\section{References}

1. Illes, S. More than a drainage fluid: the role of CSF in signaling in the brain and other effects on brain tissue. in Handbook of Clinical Neurology (eds. Deisenhammer, F., Teunissen, C. E. \& Tumani, H.) vol. 146 33-46, https://doi.org/10.1016/B978-0-12-804279-3.00003-4 (Elsevier, 2018).

2. Reiber, H. Dynamics of brain-derived proteins in cerebrospinal fluid. Clin. Chim. Acta 310, 173-186, https://doi.org/10.1016/S0009-8981(01)00573-3 (2001).

3. Lindsey, B. W. \& Tropepe, V. A comparative framework for understanding the biological principles of adult neurogenesis. Prog. Neurobiol. 80, 281-307, https://doi.org/10.1016/j.pneurobio.2006.11.007 (2006).

4. Villeda, S. A. et al. The ageing systemic milieu negatively regulates neurogenesis and cognitive function. Nature 477, 90-94, https://doi-org /10.1038/nature10357 (2011).

5. Bachy, I., Kozyraki, R. \& Wassef, M. The particles of the embryonic cerebrospinal fluid: How could they influence brain development? Brain Res. Bull. 75, 289-294, https://doi.org/10.1016/j.brainresbull.2007.10.010 (2008).

6. Zappaterra, M. W. \& Lehtinen, M. K. The cerebrospinal fluid: regulator of neurogenesis, behavior, and beyond. Cell. Mol. Life Sci. 69, 2863-2878, https://doi-org /10.1007/s00018-012-0957-x (2012).

7. Fame, R. M. \& Lehtinen, M. K. Emergence and Developmental Roles of the Cerebrospinal Fluid System. Dev. Cel/ 52, 261-275, https://doi.org/10.1016/j.devcel.2020.01.027 (2020).

8. Sawamoto, K. et al. New Neurons Follow the Flow of Cerebrospinal Fluid in the Adult Brain. Science 311, 629-632, https://doi.org/10.1126/science.1119133 (2006).

9. Lehtinen, M. K. et al. The Cerebrospinal Fluid Provides a Proliferative Niche for Neural Progenitor Cells. Neuron 69, 893-905, https://doi.org/10.1016/j.neuron.2011.01.023 (2011). 
10. Parada, C., Gato, A., Aparicio, M. \& Bueno, D. Proteome analysis of chick embryonic cerebrospinal fluid. Proteomics 6, 312-320, https://doi-org /10.1002/pmic.200500085

(2006).

11. Parada, C., Gato, Á. \& Bueno, D. Mammalian Embryonic Cerebrospinal Fluid Proteome Has Greater Apolipoprotein and Enzyme Pattern Complexity than the Avian Proteome. J. Proteome Res. 4, 24202428, https://doi-org/10.1021/pr050213t (2005).

12. Zappaterra, M. D. et al. A Comparative Proteomic Analysis of Human and Rat Embryonic Cerebrospinal Fluid. J. Proteome Res. 6, 3537-3548, https://doi-org/10.1021/pr070247w (2007).

13. Smith, J. S. et al. Characterization of individual mouse cerebrospinal fluid proteomes. Proteomics 14, 1102-1106, https://doi-org/10.1002/pmic.201300241 (2014).

14. Macron, C., Lane, L., Núñez Galindo, A. \& Dayon, L. Deep Dive on the Proteome of Human Cerebrospinal Fluid: A Valuable Data Resource for Biomarker Discovery and Missing Protein Identification. J. Proteome Res. 17, 4113-4126, https://doi-org/10.1021/acs.jproteome.8b00300 (2018).

15. Macron, C. et al. Exploration of human cerebrospinal fluid: A large proteome dataset revealed by trapped ion mobility time-of-flight mass spectrometry. Data Brief 31, 105704, https://doi.org/10.1016/j.dib.2020.105704 (2020).

16. Begcevic, I., Brinc, D., Drabovich, A. P., Batruch, I. \& Diamandis, E. P. Identification of brain-enriched proteins in the cerebrospinal fluid proteome by LC-MS/MS profiling and mining of the Human Protein Atlas. Clin. Proteomics 13, 11, https://doi.org/10.1186/s12014-016-9111-3 (2016).

17. Barker, J. M., Boonstra, R. \& Wojtowicz, J. M. From pattern to purpose: how comparative studies contribute to understanding the function of adult neurogenesis. Eur. J. Neurosci. 34, 963-977, https://doi-org/10.1111/j.1460-9568.2011.07823.x (2011).

18. Barnea, A. \& Pravosudov, V. Birds as a model to study adult neurogenesis: bridging evolutionary, comparative and neuroethological approaches. Eur. J. Neurosci. 34, 884-907, https://doiorg/10.1111/j.1460-9568.2011.07851.x (2011).

19. Patel, V. J. et al. A Comparison of Labeling and Label-Free Mass Spectrometry-Based Proteomics Approaches. J. Proteome Res. 8, 3752-3759, https://doi-org/10.1021/pr900080y (2009).

20. Zhang, G. et al. Comparative genomics reveals insights into avian genome evolution and adaptation. Science 346, 1311-1320, https://doi-org/10.1126/science.1251385 (2014).

21. Clayton, N. S. \& Emery, N. J. Avian Models for Human Cognitive Neuroscience: A Proposal. Neuron 86, 1330-1342, https://doi.org/10.1016/j.neuron.2015.04.024 (2015).

22. Olkowicz, S. et al. Birds have primate-like numbers of neurons in the forebrain. Proc. Natl. Acad. Sci. 113, 7255-7260, https://doi.org/10.1073/pnas.1517131113 (2016).

23. Němec, P. \& Osten, P. The evolution of brain structure captured in stereotyped cell count and cell type distributions. Curr. Opin. Neurobiol. 60, 176-183, https://doi.org/10.1016/j.conb.2019.12.005 (2020). 
24. Wirthlin, M. et al. Parrot Genomes and the Evolution of Heightened Longevity and Cognition. Curr. Biol. 28, 4001-4008.e7, https://doi.org/10.1016/j.cub.2018.10.050 (2018).

25. Iwaniuk, A. N., Dean, K. M. \& Nelson, J. E. Interspecific Allometry of the Brain and Brain Regions in Parrots (Psittaciformes): Comparisons with Other Birds and Primates. Brain. Behav. Evol. 65, 40-59, https://doi-org/10.1159/000081110 (2005).

26. Auersperg, A. M. I., Szabo, B., von Bayern, A. M. P. \& Bugnyar, T. Object permanence in the Goffin cockatoo (Cacatua goffini). J. Comp. Psychol. 128, 88-98, https://doi.org/10.1037/a0033272 (2014).

27. Pepperberg, I. M., Willner, M. R. \& Gravitz, L. B. Development of Piagetian object permanence in grey parrot (Psittacus erithacus). J. Comp. Psychol. 111, 63-75, https://doi.org/10.1037/07357036.111.1.63 (1997).

28. Emery, N. J. \& Clayton, N. S. Evolution of the avian brain and intelligence. Curr. Biol. 15, R946-R950, https://doi.org/10.1016/j.cub.2005.11.029 (2005).

29. Péron, F., Rat-Fischer, L., Lalot, M., Nagle, L. \& Bovet, D. Cooperative problem solving in African grey parrots (Psittacus erithacus). Anim. Cogn. 14, 545-553, https://doi-org/10.1007/s10071-011-0389-2 (2011).

30. Hobson, E. A., Avery, M. L. \& Wright, T. F. The socioecology of Monk Parakeets: Insights into parrot social complexity. Auk Ornithol. Adv. 131, 756-775, https://doi-org/10.1642/AUK-14-14.1 (2014).

31. Brauth, S. E., Heaton, J. T., Shea, S. D., Durand, S. E. \& Hall, W. S. Functional Anatomy of Forebrain Vocal Control Pathways in the Budgerigar (Melopsittacus undulatus)a. Ann. N. Y. Acad. Sci. 807, 368-385, https://doi-org/10.1111/j.1749-6632.1997.tb51933.x (1997).

32. Pepperberg, I. M. The Alex Studies: cognitive and communicative abilities of grey parrots. (Harvard University Press, 2009).

33. Emery, N. J. Cognitive ornithology: the evolution of avian intelligence. Philos. Trans. R. Soc. B Biol. Sci. 361, 23-43, https://doi-org/10.1098/rstb.2005.1736 (2006).

34. Güntürkün, O. \& Bugnyar, T. Cognition without Cortex. Trends Cogn. Sci. 20, 291-303, https://doi.org/10.1016/j.tics.2016.02.001 (2016).

35. Lambert, M. L., Jacobs, I., Osvath, M. \& Bayern, A. M. P. von. Birds of a feather? Parrot and corvid cognition compared. Behaviour 156, 505-594, https://doi.org/10.1163/1568539X-00003527 (2019).

36. Iwaniuk, A. N. \& Nelson, J. E. Developmental differences are correlated with relative brain size in birds: a comparative analysis. Can. J. Zool. 81, 1913-1928, https://doi.org/10.1139/z03-190 (2003).

37. Munshi-South, J. \& Wilkinson, G. S. Diet Influences Life Span in Parrots (Psittaciformes). The Auk 123, 108-118, https://doi-org/10.1093/auk/123.1.108 (2006).

38. Provost, K. L., Joseph, L. \& Smith, B. T. Resolving a phylogenetic hypothesis for parrots: implications from systematics to conservation. Emu - Austral Ornithol. 118, 7-21, https://doiorg/10.1080/01584197.2017.1387030 (2018). 
39. Wright, J. C., Beynon, R. J. \& Hubbard, S. J. Cross Species Proteomics. in Proteome Bioinformatics (eds. Hubbard, S. J. \& Jones, A. R.) 123-135, https://doi-org/10.1007/978-1-60761-444-9_9 (Humana Press, 2010).

40. Liska, A. J. \& Shevchenko, A. Expanding the organismal scope of proteomics: Cross-species protein identification by mass spectrometry and its implications. Proteomics 3, 19-28, https://doiorg.ezproxy.is.cuni.cz/10.1002/pmic.200390004 (2003).

41. Dayon, L. et al. Proteomes of Paired Human Cerebrospinal Fluid and Plasma: Relation to BloodBrain Barrier Permeability in Older Adults. J. Proteome Res. 18, 1162-1174, https://doi-org /10.1021/acs.jproteome.8b00809 (2019).

42. Guldbrandsen, A. et al. In-depth characterization of the cerebrospinal fluid (CSF) proteome displayed through the CSF proteome resource (CSF-PR). Mol. Cell. Proteomics MCP 13, 3152-3163, https://doi.org/10.1074/mcp.M114.038554 (2014).

43. Thompson, E. J. CHAPTER 4 - Differences between proteins in CSF and serum. in Proteins of the Cerebrospinal Fluid (ed. Thompson, E. J.) 33-41 (Academic Press, 2005).

44. Reiber, H. Proteins in cerebrospinal fluid and blood: barriers, CSF flow rate and source-related dynamics. Restor. Neurol. Neurosci. 21, 79-96 (2003).

45. Anderson, N. L. \& Anderson, N. G. The Human Plasma Proteome: History, Character, and Diagnostic Prospects. Mol. Cell. Proteomics 1, 845-867, https://doi.org/10.1074/mcp.R200007-MCP200 (2002).

46. Aaseb $\varnothing$, E. et al. Effects of blood contamination and the rostro-caudal gradient on the human cerebrospinal fluid proteome. PloS One 9, e90429, https://doi.org/10.1371/journal.pone.0090429 (2014).

47. You, J.-S. et al. The impact of blood contamination on the proteome of cerebrospinal fluid. Proteomics 5, 290-296, https://doi-org/10.1002/pmic.200400889 (2005).

48. Zhang, C. Proteomic Studies on the Development of the Central Nervous System and Beyond. Neurochem. Res. 35, 1487-1500, https://doi-org/10.1007/s11064-010-0218-z (2010).

49. Macron, C., Lane, L., Núñez Galindo, A. \& Dayon, L. Identification of Missing Proteins in Normal Human Cerebrospinal Fluid. J. Proteome Res. 17, 4315-4319, https://doiorg/10.1021/acs.jproteome.8b00194 (2018).

50. Kroksveen, A. C. et al. In-Depth Cerebrospinal Fluid Quantitative Proteome and Deglycoproteome Analysis: Presenting a Comprehensive Picture of Pathways and Processes Affected by Multiple Sclerosis. J. Proteome Res. 16, 179-194, https://doi-org/10.1021/acs.jproteome.6b00659 (2017).

51. Nilsson, C. et al. Circadian variation in human cerebrospinal fluid production measured by magnetic resonance imaging. Am. J. Physiol.-Regul. Integr. Comp. Physiol. 262, R20-R24, https://doi.org/10.1152/ajpregu.1992.262.1.R20 (1992).

52. Lehtinen, M. K. \& Walsh, C. A. Neurogenesis at the Brain-Cerebrospinal Fluid Interface. Annu. Rev. Cell Dev. Biol. 27, 653-679, https://doi.org/10.1146/annurev-cellbio-092910-154026 (2011). 
53. Martín, C. et al. FGF2 plays a key role in embryonic cerebrospinal fluid trophic properties over chick embryo neuroepithelial stem cells. Dev. Biol. 297, 402-416, https://doi.org/10.1016/j.ydbio.2006.05.010 (2006).

54. Salehi, Z., Mashayekhi, F., Naji, M. \& Pandamooz, S. Insulin-like growth factor-1 and insulin-like growth factor binding proteins in cerebrospinal fluid during the development of mouse embryos. J. Clin. Neurosci. 16, 950-953, https://doi.org/10.1016/j.jocn.2008.09.018 (2009).

55. Buddensiek, J., Dressel, A., Kowalski, M., Storch, A. \& Sabolek, M. Adult cerebrospinal fluid inhibits neurogenesis but facilitates gliogenesis from fetal rat neural stem cells. J. Neurosci. Res. 87, 30543066, https://doi-org/10.1002/jnr.22150 (2009).

56. Buddensiek, J. et al. Cerebrospinal fluid promotes survival and astroglial differentiation of adult human neural progenitor cells but inhibits proliferation and neuronal differentiation. BMC Neurosci. 11, 48, https://doi-org/10.1186/1471-2202-11-48 (2010).

57. Gato, A., Alonso, M. I., Lamus, F. \& Miyan, J. Neurogenesis: A process ontogenically linked to brain cavities and their content, CSF. Semin. Cell Dev. Biol. 102, 21-27, https://doi.org/10.1016/j.semcdb.2019.11.008 (2020).

58. Alvarez-Buylla, A. \& Nottebohm, F. Migration of young neurons in adult avian brain. Nature 335, 353354, https://doi-org/10.1038/335353a0 (1988).

59. Goldman, S. A. \& Nottebohm, F. Neuronal production, migration, and differentiation in a vocal control nucleus of the adult female canary brain. Proc. Natl. Acad. Sci. U. S. A. 80, 2390-2394, https://doi.org/10.1073/pnas.80.8.2390 (1983).

60. Patel, S. N., Clayton, N. S. \& Krebs, J. R. Spatial learning induces neurogenesis in the avian brain. Behav. Brain Res. 89, 115-128, https://doi.org/10.1016/S0166-4328(97)00051-X (1997).

61. Ling, C., Zuo, M., Alvarez-Buylla, A. \& Cheng, M. F. Neurogenesis in juvenile and adult ring doves. J. Comp. Neurol. 379, 300-312, https://doi-org/10.1002/(SICI)1096-9861(19970310)379:2<300::AIDCNE10>3.0.CO;2-T (1997).

62. Mezey, S. et al. Postnatal changes in the distribution and density of neuronal nuclei and doublecortin antigens in domestic chicks (Gallus domesticus). J. Comp. Neurol. 520, 100-116, https://doiorg/10.1002/cne.22696 (2012).

63. Melleu, F. F., Santos, T. S., Lino-de-Oliveira, C. \& Marino-Neto, J. Distribution and characterization of doublecortin-expressing cells and fibers in the brain of the adult pigeon (Columba livia). J. Chem. Neuroanat. 47, 57-70, https://doi.org/10.1016/j.jchemneu.2012.10.006 (2013).

64. Mazengenya, P., Bhagwandin, A., Nkomozepi, P., Manger, P. R. \& Ihunwo, A. O. Putative adult neurogenesis in two domestic pigeon breeds (Columba livia domestica): racing homer versus utility carneau pigeons. Neural Regen. Res. 12, 1086-1096, https://doi.org/10.4103/1673-5374.211187 (2017).

65. Mazengenya, P., Bhagwandin, A., Manger, P. R. \& Ihunwo, A. O. Putative Adult Neurogenesis in Old World Parrots: The Congo African Grey Parrot (Psittacus erithacus) and Timneh Grey Parrot (Psittacus timneh). Front. Neuroanat. 12, 7, https://doi.org/10.3389/fnana.2018.00007 (2018). 
66. Mazengenya, P., Bhagwandin, A. \& Ihunwo, A. O. Putative adult neurogenesis in palaeognathous birds: The common ostrich (Struthio camelus) and emu (Dromaius novaehollandiae). Int. J. Dev. Neurosci. 80, 613-635, https://doi-org/10.1002/jdn.10057 (2020).

67. Doetsch, F. \& Scharff, C. Challenges for Brain Repair: Insights from Adult Neurogenesis in Birds and Mammals. Brain. Behav. Evol. 58, 306-322, https://doi-org/10.1159/000057572 (2001).

68. Anderson, D. K. \& Hazelwood, R. L. Chicken cerebrospinal fluid: normal composition and response to insulin administration. J. Physiol. 202, 83-95, https://doi.org/10.1113/jphysiol.1969.sp008796 (1969).

69. Černá, M., Kuntová, B., Talacko, P., Stopková, R. \& Stopka, P. Differential regulation of vaginal lipocalins (OBP, MUP) during the estrous cycle of the house mouse. Sci. Rep. 7, 1-10, https://doiorg/10.1038/s41598-017-12021-2 (2017).

70. Cox, J. et al. Accurate Proteome-wide Label-free Quantification by Delayed Normalization and Maximal Peptide Ratio Extraction, Termed MaxLFQ. Mol. Cell. Proteomics MCP 13, 2513-2526, https://doi.org/10.1074/mcp.M113.031591 (2014).

71. Rodriguez, J., Gupta, N., Smith, R. D. \& Pevzner, P. A. Does Trypsin Cut Before Proline? J. Proteome Res. 7, 300-305, https://doi-org/10.1021/pr0705035 (2008).

72. Perez-Riverol, Y. et al. The PRIDE database and related tools and resources in 2019: improving support for quantification data. Nucleic Acids Res. 47, D442-D450, https://doi-org /10.1093/nar/gky1106 (2019).

73. Hillier, L. W. et al. Sequence and comparative analysis of the chicken genome provide unique perspectives on vertebrate evolution. Nature 432, 695-716, https://doi.org/10.1038/nature03154 (2004).

74. Ganapathy, G. et al. High-coverage sequencing and annotated assemblies of the budgerigar genome. GigaScience 3, 11, https://doi-org/10.1186/2047-217X-3-11 (2014).

75. Kriventseva, E. V. et al. OrthoDB v10: sampling the diversity of animal, plant, fungal, protist, bacterial and viral genomes for evolutionary and functional annotations of orthologs. Nucleic Acids Res. 47, D807-D811, https://doi-org/10.1093/nar/gky1053 (2019).

76. Thomas, P. D. et al. PANTHER: A Library of Protein Families and Subfamilies Indexed by Function. Genome Res. 13, 2129-2141, https://doi-org/10.1101/gr.772403 (2003).

77. Ashburner, M. et al. Gene Ontology: tool for the unification of biology. Nat. Genet. 25, 25-29, https://doi-org/10.1038/75556 (2000).

78. Reimand, J. et al. g:Profiler-a web server for functional interpretation of gene lists (2016 update). Nucleic Acids Res. 44, W83-W89, https://doi-org/10.1093/nar/gkw199 (2016).

79. Kanehisa, M., Furumichi, M., Tanabe, M., Sato, Y. \& Morishima, K. KEGG: new perspectives on genomes, pathways, diseases and drugs. Nucleic Acids Res. 45, D353-D361, https://doiorg/10.1093/nar/gkw1092 (2017).

80. Fabregat, A. et al. The Reactome Pathway Knowledgebase. Nucleic Acids Res. 46, D649-D655, https://doi-org/10.1093/nar/gkx1132 (2018). 
81. R Development Core Team. R: The R Project for Statistical Computing. https://www.r-project.org/ (2015).

82. Hulsen, T., de Vlieg, J. \& Alkema, W. BioVenn - a web application for the comparison and visualization of biological lists using area-proportional Venn diagrams. BMC Genomics 9, 488, https://doi.org/10.1186/1471-2164-9-488 (2008).

83. Bardou, P., Mariette, J., Escudié, F., Djemiel, C. \& Klopp, C. Jvenn: an interactive Venn diagram viewer. BMC Bioinformatics 15, 293, https://doi.org/10.1186/1471-2105-15-293 (2014).

84. Huber, W., von Heydebreck, A., Sültmann, H., Poustka, A. \& Vingron, M. Variance stabilization applied to microarray data calibration and to the quantification of differential expression. Bioinformatics 18, S96-S104, https://doi-org/10.1093/bioinformatics/18.suppl_1.S96 (2002).

85. Välikangas, T., Suomi, T. \& Elo, L. L. A systematic evaluation of normalization methods in quantitative label-free proteomics. Brief. Bioinform. 19, 1-11, https://doi-org/10.1093/bib/bbw095 (2016).

86. von Mering, C. et al. STRING: a database of predicted functional associations between proteins. Nucleic Acids Res. 31, 258-261, https://doi-org/10.1093/nar/gkg034 (2003).

87. Ge, S. X., Jung, D. \& Yao, R. ShinyGO: a graphical gene-set enrichment tool for animals and plants. Bioinformatics 36, 2628-2629, https://doi-org/10.1093/bioinformatics/btz931 (2020).

88. Subramanian, A. et al. Gene set enrichment analysis: A knowledge-based approach for interpreting genome-wide expression profiles. Proc. Natl. Acad. Sci. 102, 15545-15550, https://doi.org/10.1073/pnas.0506580102 (2005).

\section{Figures}


a
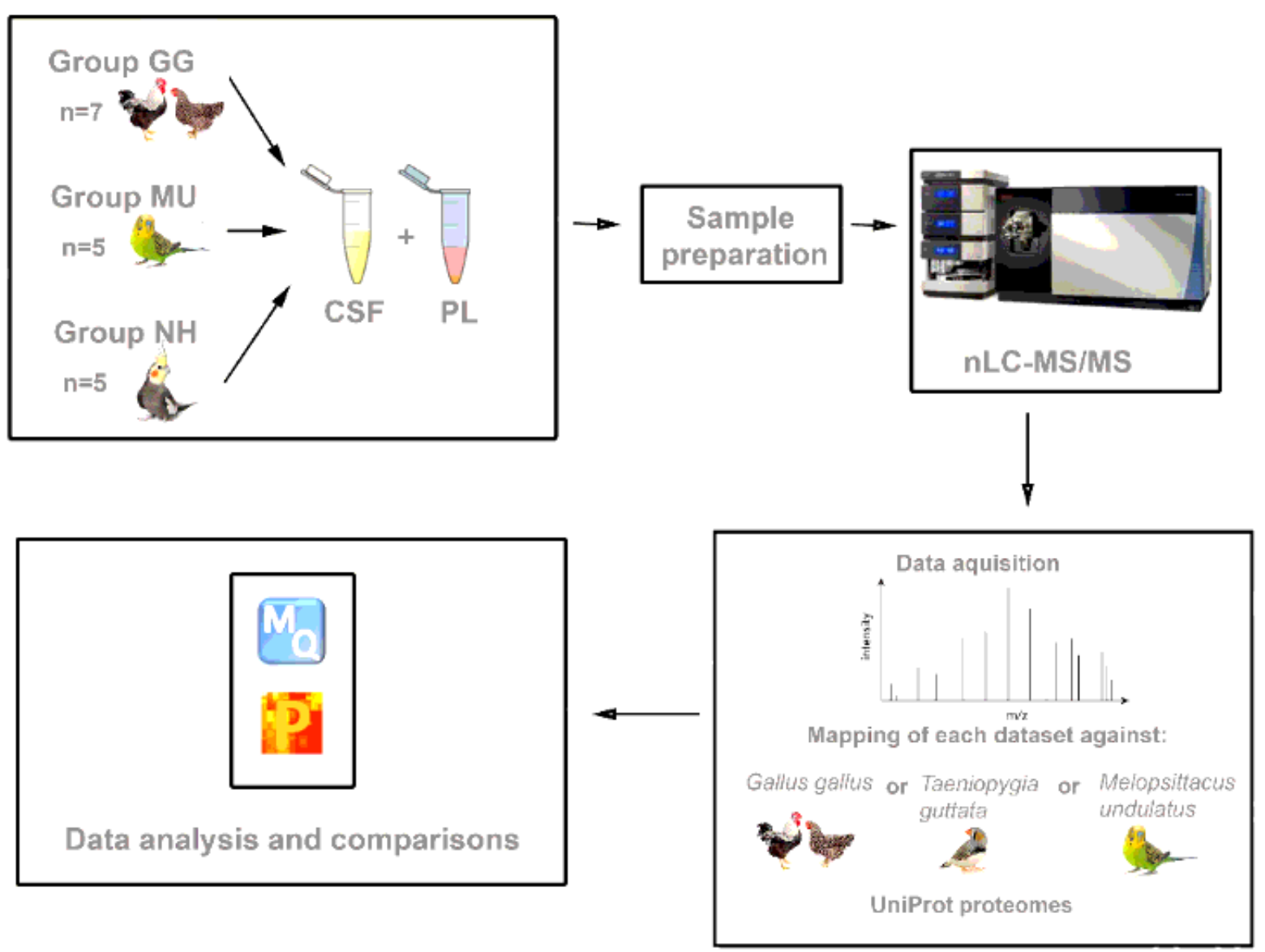

b

Cross-species protein identification

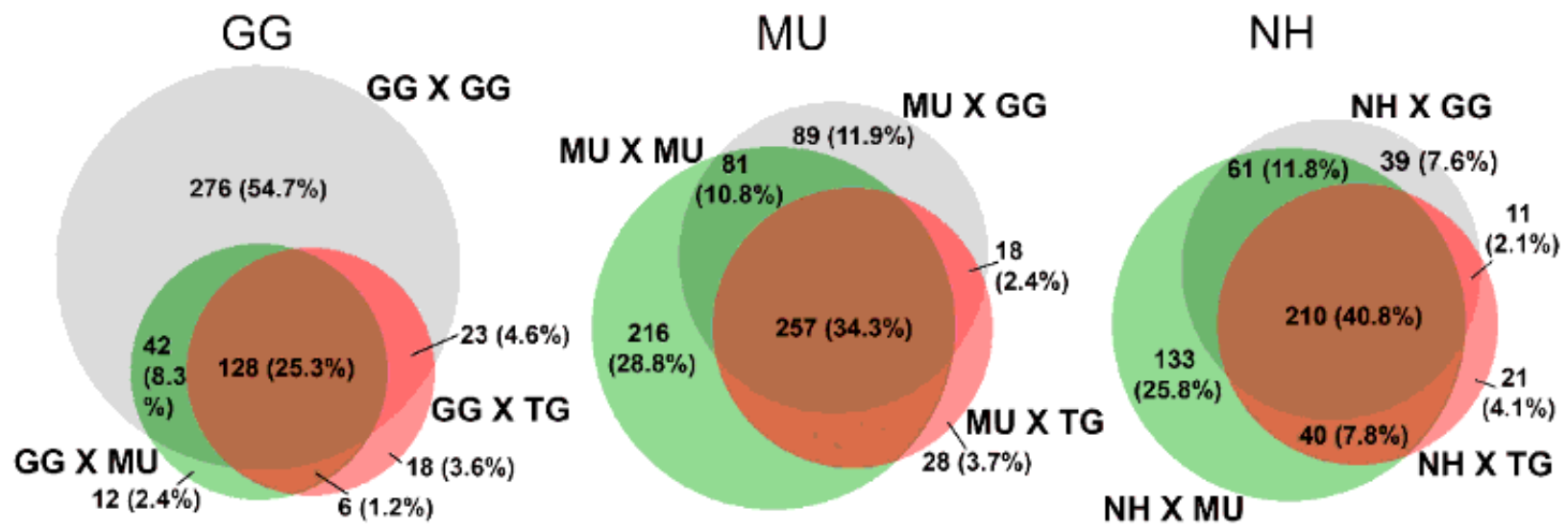

\section{Figure 1}

Comparison of the nLC-MS/MS cross-species protein identification success in both cerebrospinal fluid (CSF) and plasma (PL) samples of chickens (GG), budgerigars (MU) and cockatiels (NH), after mapping to three selected avian reference proteomes GG, MU and Taeniopygia guttata (TG). (a) The overview of the study design and mapping approach is schematically illustrated. (b) Venn diagrams show the 
proportions of proteins identified based on the different reference proteomes of GG (grey), TG (red) and $\mathrm{MU}$ (green) for GG, MU and $\mathrm{NH}$.

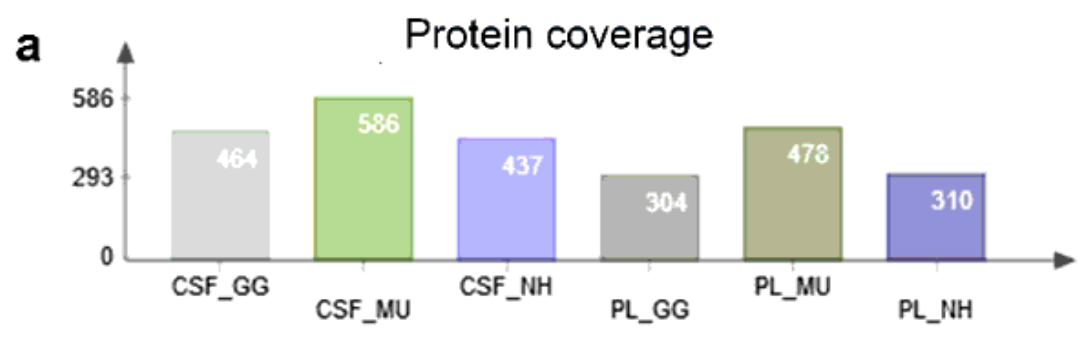

Number of elements: specific (1) or shared by 2, 3, ... lists

\begin{tabular}{|c||c|c|c|c|c|}
\hline 109 & $\sqrt{127}$ & 82 & 381 & 204 \\
\hline 6 & $5(41)$ & 3 & 2 & 1
\end{tabular}

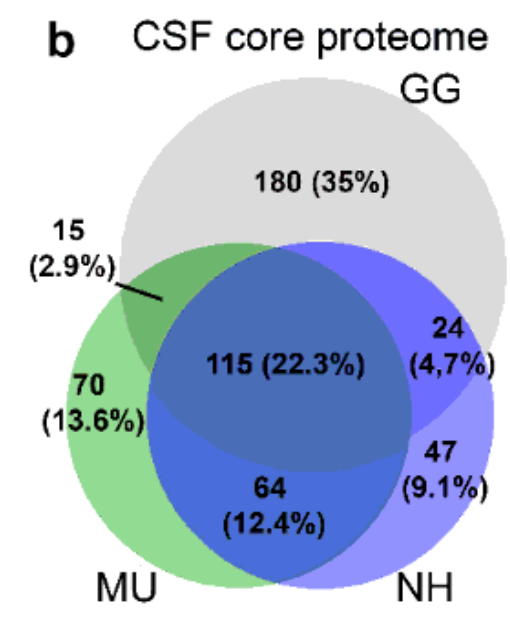

C PL core proteome

d Shared proteins
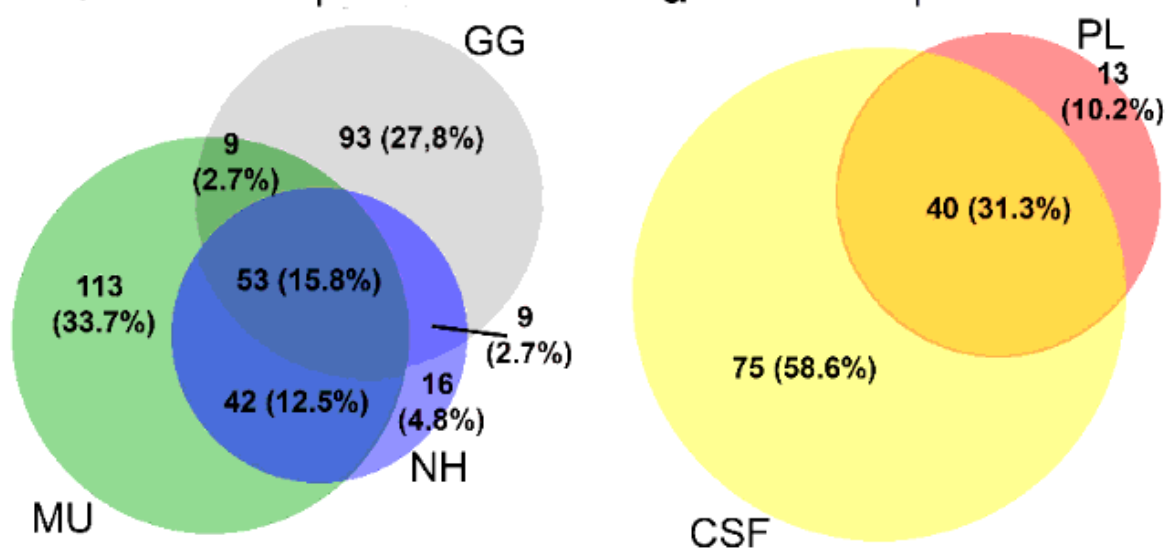

Figure 2

Overview of the proteomic coverage across species and body fluids. The identified plasma (PL) and cerebrospinal fluid (CSF) proteins of chicken (GG), budgerigar (MU) and cockatiel (NH) and the shared identifications between the groups are demonstrated in (a). The Venn diagrams are showing the overlapping numbers and percentages of core proteomes of CSF (b) and PL (c) for GG, MU and NH, and (d) the numbers and percentages of shared proteins across all the three species for CSF and PL. 

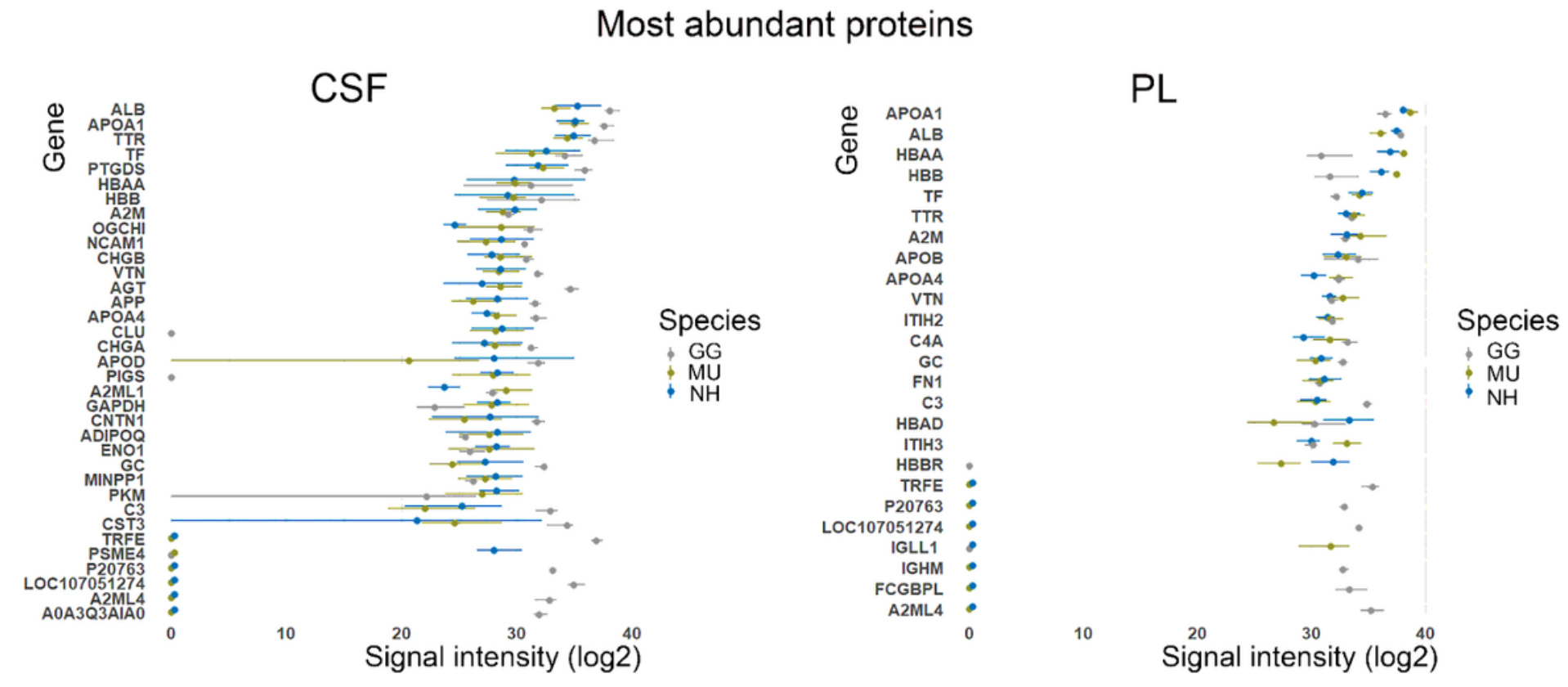

Figure 3

The top 2\% most abundant cerebrospinal fluid (CSF) and plasma (PL) proteins of chicken (GG), budgerigar $(\mathrm{MU})$ and cockatiel $(\mathrm{NH})$ are presented using their gene code: $\mathrm{GG}$ - grey, $\mathrm{MU}$ - green and $\mathrm{NH}$ blue. The logarithmic normalised abundances are shown as a range (minimum-maximum) across all individuals ( $G G n=7, M U n=5, N H n=5)$ with a mean highlighted as a dot. 
a

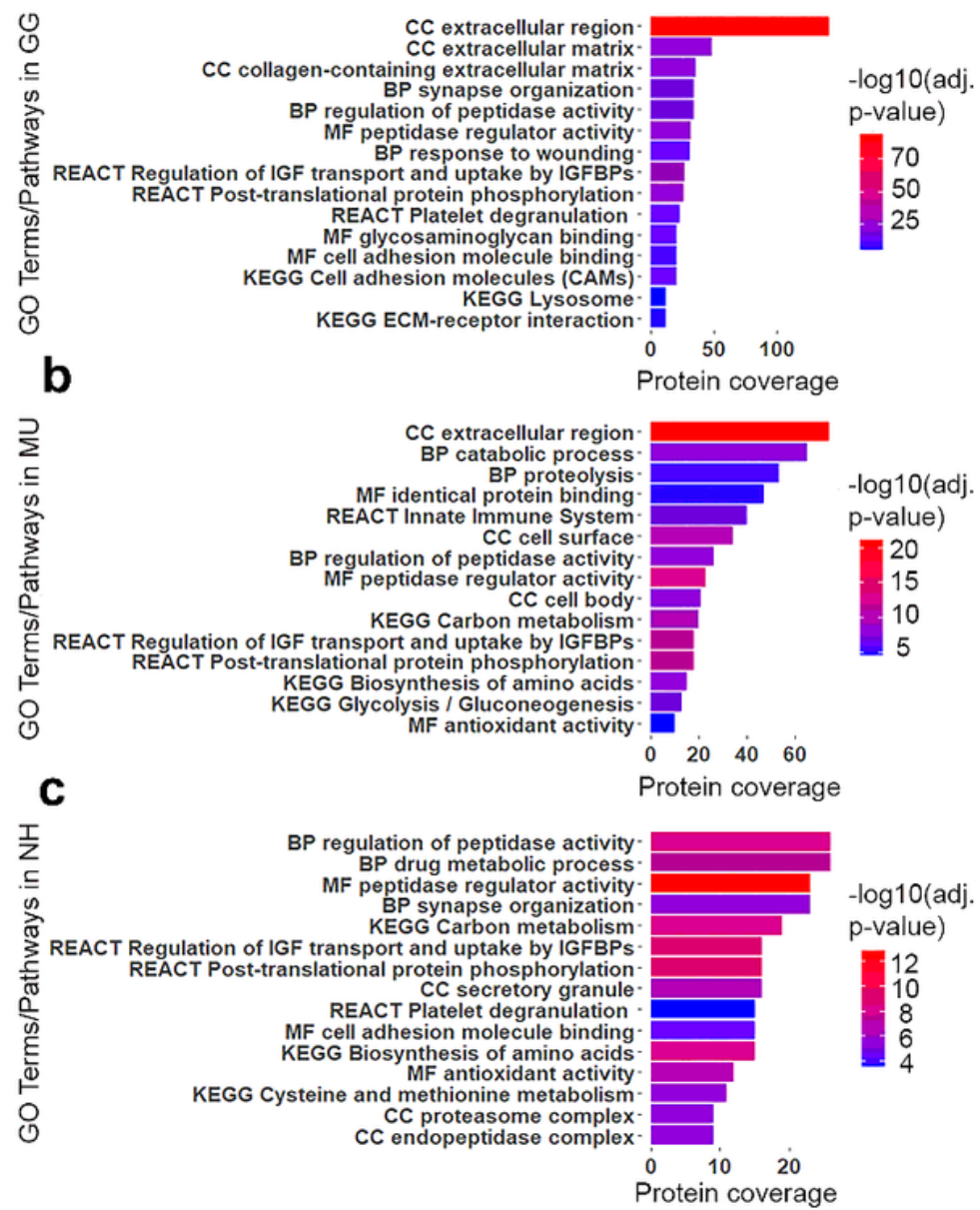

Over-represented terms in PL
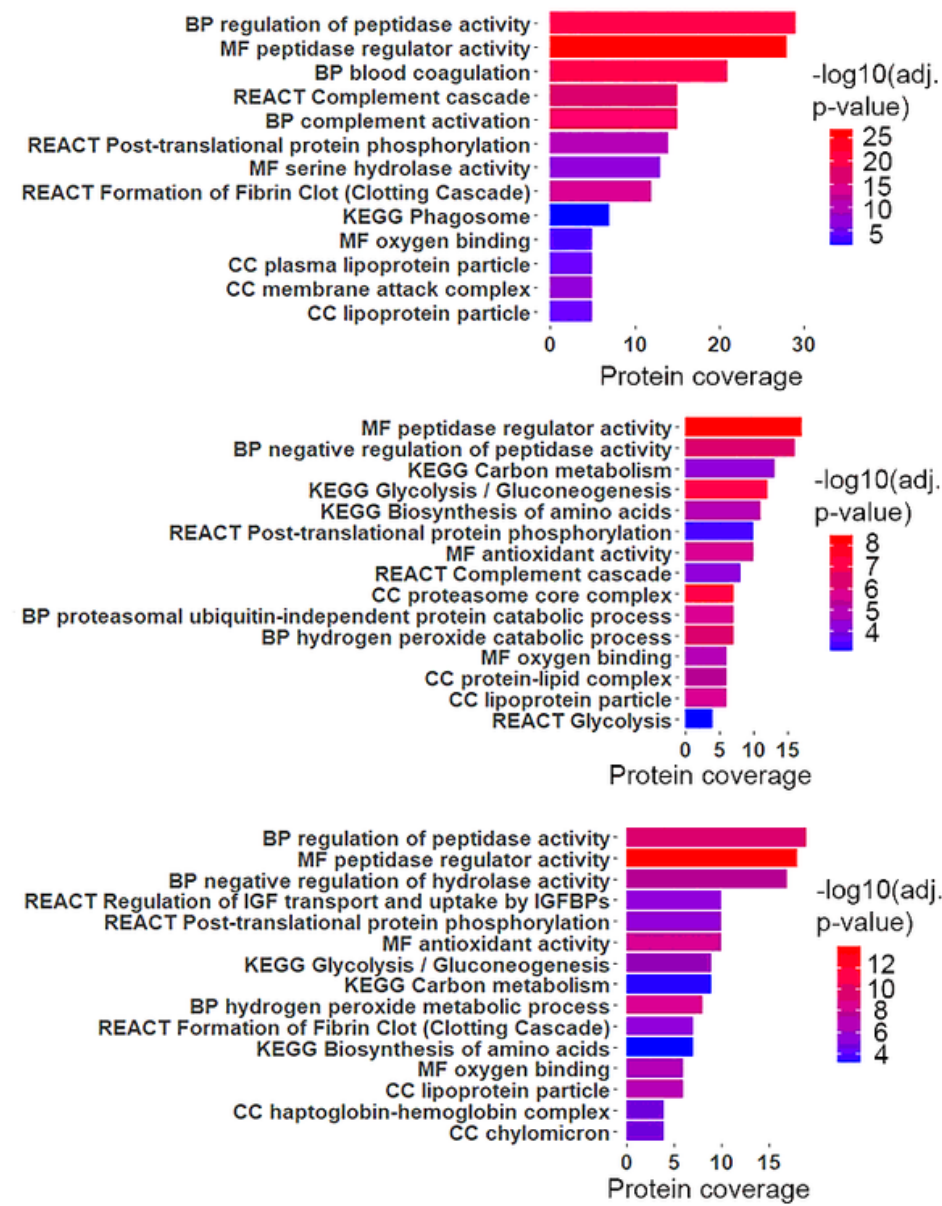

\section{Figure 4}

Top significant over-represented pathways revealed after functional enrichment analysis of the cerebrospinal fluid (CSF) and blood plasma (PL). Enriched pathways in CSF are shown on the left-hand side and in PL on the right-hand side for (a) chicken (GG), (b) budgerigar (MU) and (c) cockatiel (NH). The $\mathrm{X}$-axis indicates the numbers of genes represented in each pathway, the y-axis indicates the GO (Molecular function, MF; Biological Process, BP; Cellular component, CC) or Pathways terms (KEGG, REACT) involved, and the colours represent the size of the negative log10 of the adjusted p-value (adjusted p<0.05). IGF Insulin-like Growth Factor; IGFBPs Insulin-like Growth Factor Binding Proteins. 

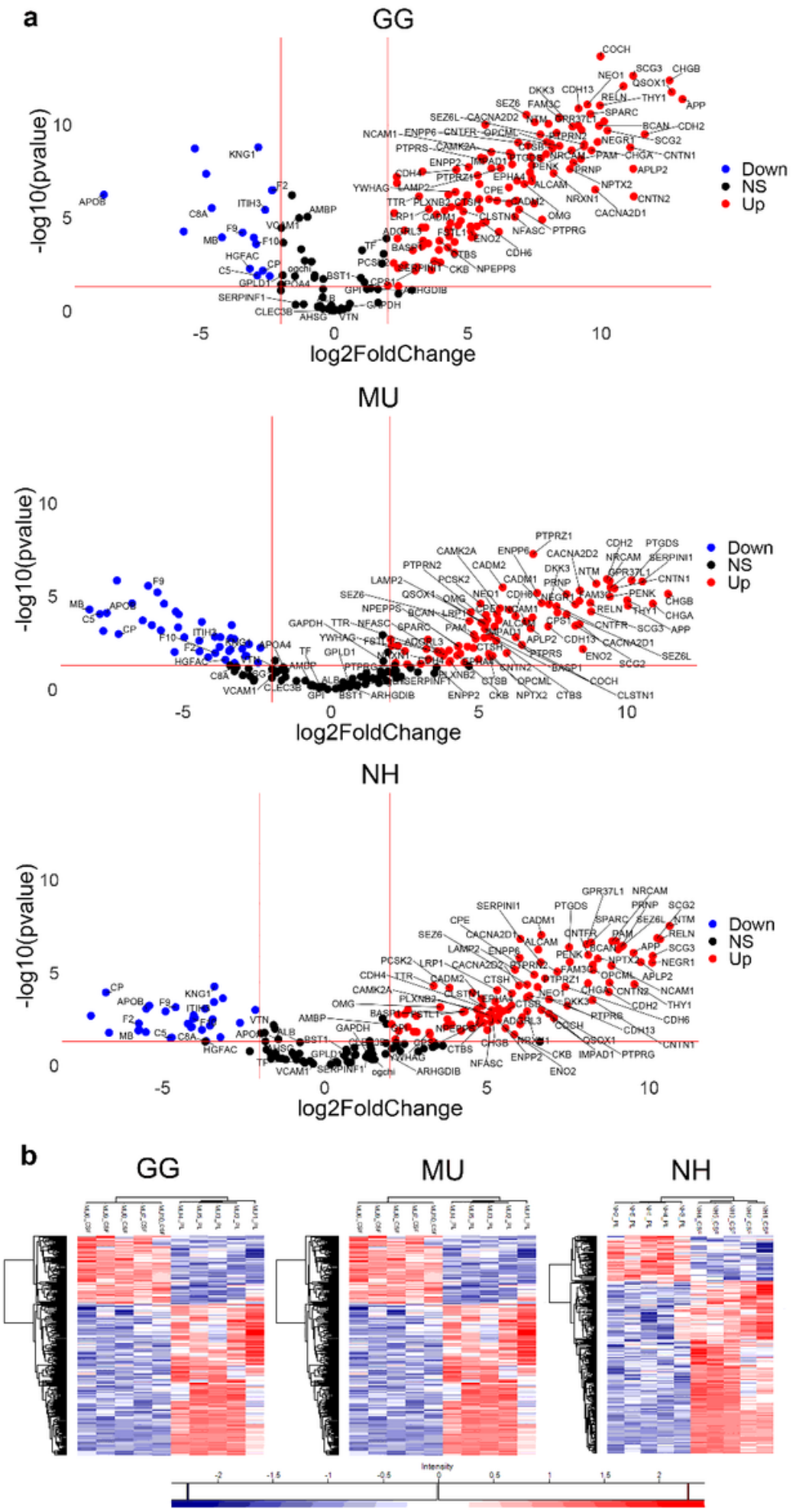

Figure 5

Comparative analyses of cerebrospinal fluid (CSF) and plasma (PL) proteomes. The distribution of the shared proteins across species and clustering of over-represented and down-represented proteins between the two fluids is shown in for chicken (GG), budgerigar (MU) and cockatiel (NH). (a) The volcano plots graphically demonstrate the fold changes on p-values between CSF and PL proteomes that are shared across species, following Student t-tests corrected by the Permutation Based false-discovery rate 
(FDR) (FDR-adjusted p-value<0.05). The fold differences of the proteins (x-axis) dependent on their $\mathrm{p}$ values (y-axis) are shown in dots, colour-coded in blue, red and black, demonstrating the significantly under-represented proteins (Down), over-represented proteins (Up), and non-significantly represented proteins (NS, $p<0.05$, fold change cut-off $\geq 2$ ) respectively in CSF compared to $P L$ for $G G, M U$ and $N H$. The proteins that are commonly differentiated are indicated and labelled using their gene codes. (b) Hierarchical clustering heatmaps show differences in protein expression between CSF and PL in GG, MU and $\mathrm{NH}$, respectively ( $<<0.05$, after multiple testing). Proteins with high (red) and low (blue) expression form two clusters differentiating the fluids. 


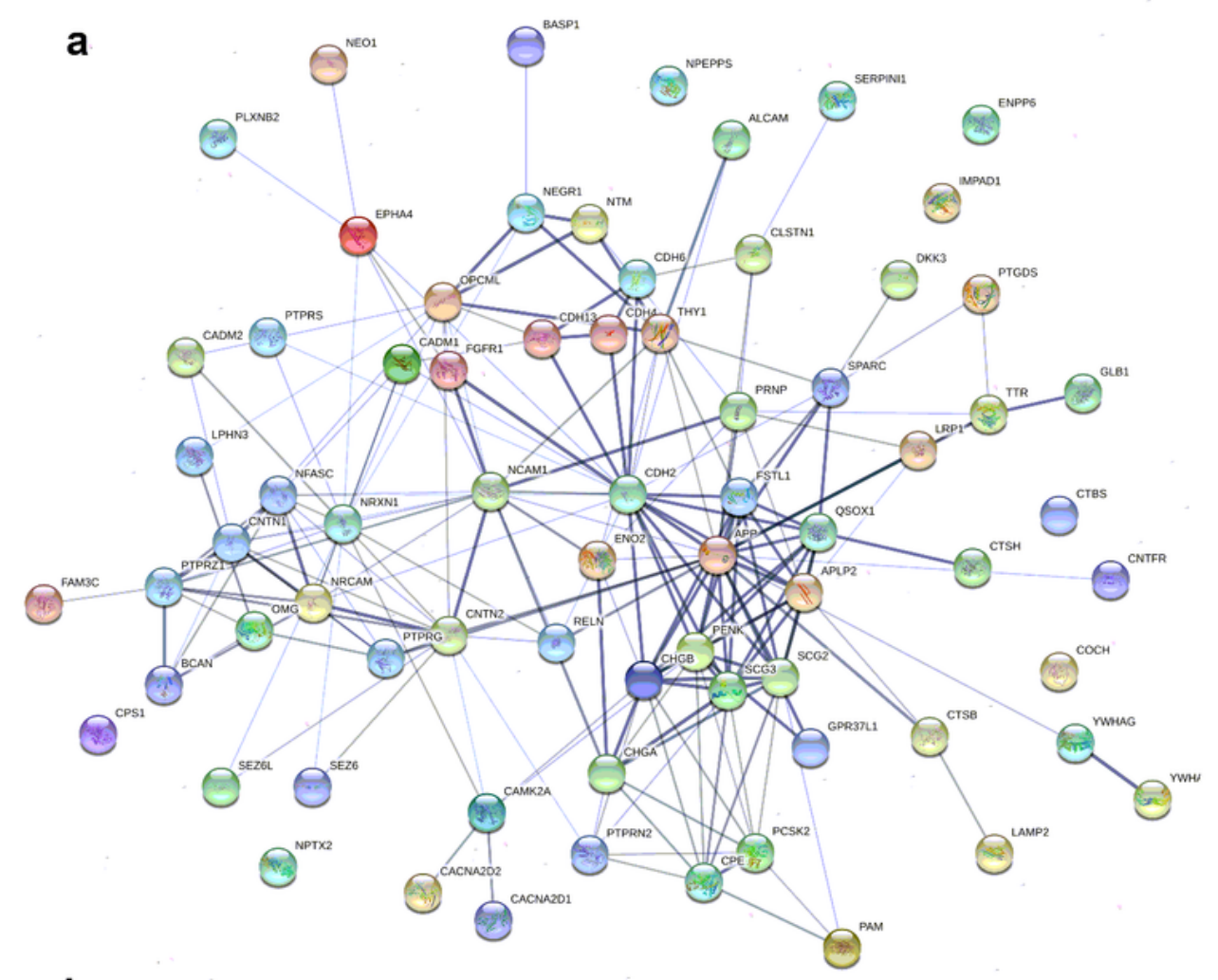

b

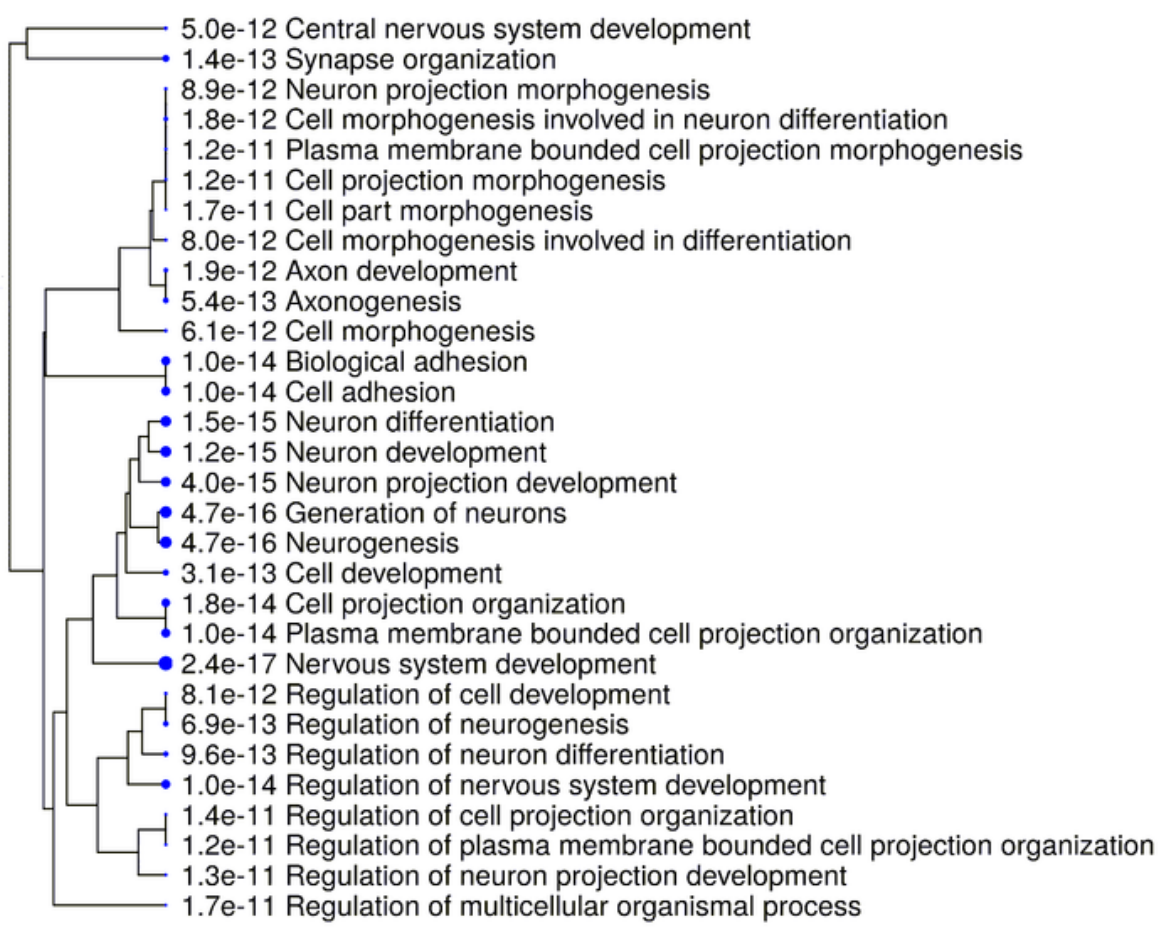

Figure 6

Protein-protein interactions (PPIs) and enriched pathways of 72 over-represented proteins in CSF compared to PL commonly in chicken, budgerigar and cockatiel. (a) STRING analysis of the protein interaction network showing associations of the proteins. Line thickness indicates the strength of data support. (b) Hierarchical clustering tree summarizing the correlation among the significant pathways enriched in the 72 commonly over-represented CSF proteins across the three species. GO terms of 
biological functions revealed pathways related to neurogenesis and neuron differentiation. Pathways with many shared genes are clustered together and dot sizes indicate the degree of statistical significance.

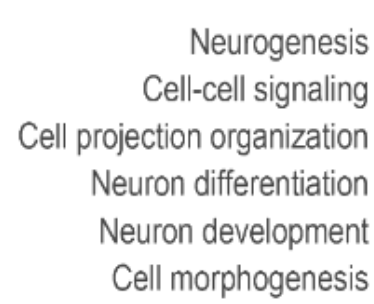

Regulation of nervous system development

Regulation of cell development

Cell-junction organization

Cell morphogenesis involved in differentiation

Axon development

Cellular component morphogenesis

Cell part morphogenesis

Regulation of cell projection organization

Head development

Cell morphogenesis involved in neuron differentiation

Nervous system process

Regulation of neuron differentiation

Synaptic signaling

Synapse organization

Regulation of neuron projection development

Regulation of cell morphogenesis

Positive regulation of nervous system development

Behaviour

Regulation of trans synaptic signaling

Regulation of cell morphogenesis involved in differentiation

Cell surface receptor signaling pathway involved in cell-cell signalling

Cell-cell adhesion via plasma membrane adhesion molecules

Neuron projection guidance

Gliogenesis

Negative regulation of cell development

Negative regulation of nervous system development

Regulation of synapse structure or activity

Glial-cell differentiation

Negative regulation of neuron differentiation

Developmental maturation

Cognition

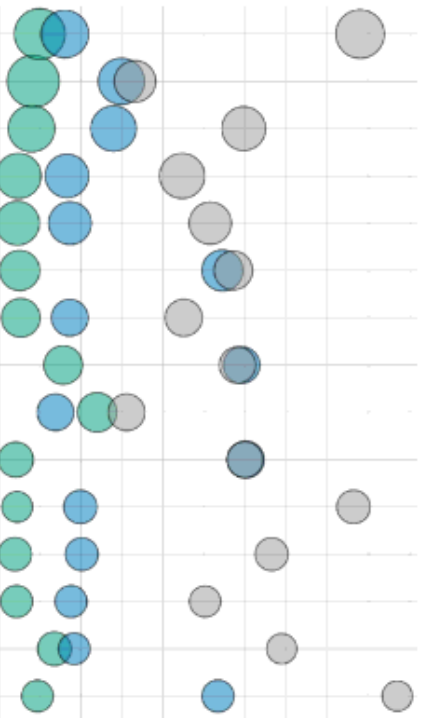

0
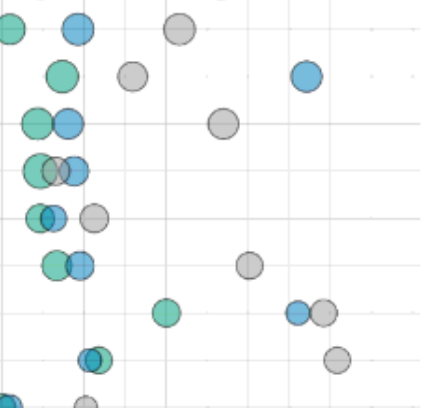

西
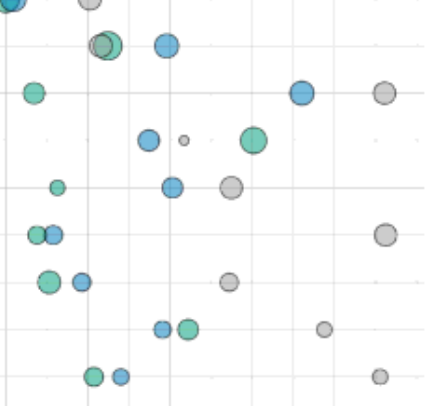

$\infty \quad 0$

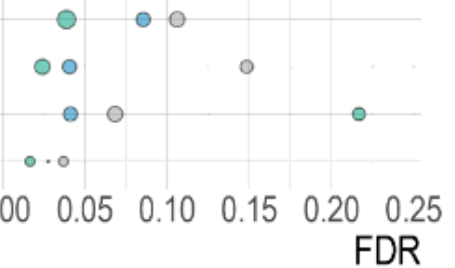

Species $\bigcirc \mathrm{GG} \bigcirc \mathrm{MU} \bigcirc \mathrm{NH}$ Genes $\circ 20 \bigcirc 40 \bigcirc 60 \bigcirc 80 \bigcirc 100$

\section{Figure 7}

Significant gene sets related to neural function commonly enriched in all species. The dot plot shows the identified enriched terms by GSEA with nominal p-value $<0.05$. P-values were adjusted for multiplicity of 
testing. The $x$-axis shows the range of the false discovery rate (FDR) values, the $y$-axis lists the GO Biological function terms, the colours indicate the involved species and the dot size shows the number of genes in each set.
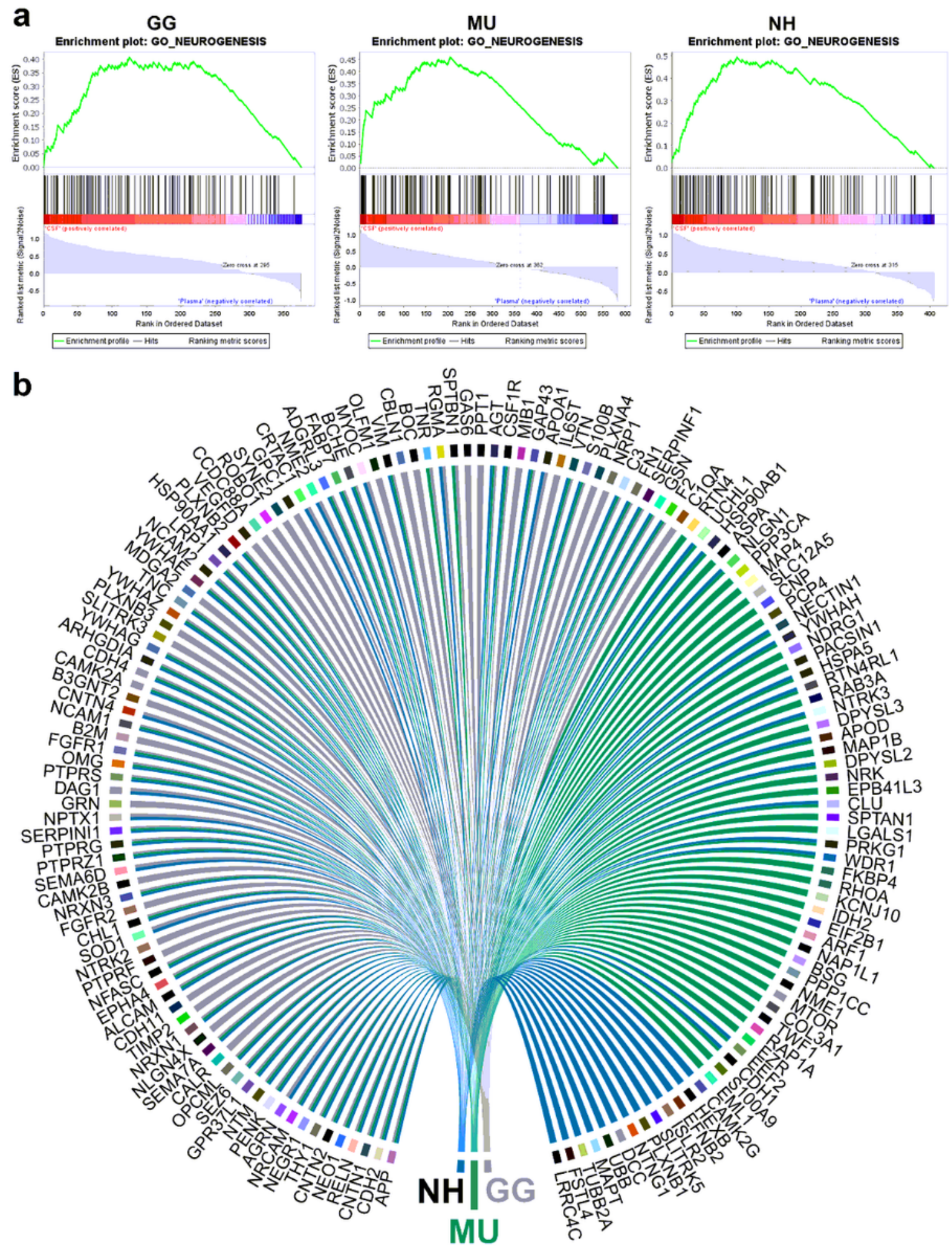

Figure 8

Gene set enrichment analysis (GSEA) and comparison of cerebrospinal fluid and plasma proteomes reveals neurogenesis in all studied avian species. (a) GSEA plots of the GO term Neurogenesis for chicken 
(GG), budgerigar (MU) and cockatiel (NH) in cerebrospinal fluid compared to plasma (b) Circular plot representation of proteins identified by GSEA with the GO term Neurogenesis for GG, MU and NH. The proteins are labelled by their gene codes.

\section{Supplementary Files}

This is a list of supplementary files associated with this preprint. Click to download.

- Supplementarymaterial.pdf

- SupplementaryTableS1.nLCMSMSoutputfromdifferentmappingapproaches.xlsx

- SupplementaryTableS2.CoreproteomelistsforCSFandPL.xIsx

- SupplementaryTableS3.GOClassificationandPathwayanalysesofCSFandPL.xIsx

- SupplementaryTableS4.DifferentiallyrepresentedproteinsinCSFvsplasma.xlsx

- SupplementaryTableS5.GSEAresults.xlsx 